\title{
THE MEMBRANE/AQUEOUS PARTITIONING AS AN ESSENTIAL TOOL FOR PHARMACOKINETIC PROFILING TO SUPPORT DRUG DESIGN
}

\author{
E.Fernandes ${ }^{1 *}$, T.Soares $^{1,2}$, A.Almeida ${ }^{3}$, B.Sarmento ${ }^{3,4}$, M.Elisabete C.D.Real Oliveira ${ }^{1}$, M.Lúcio ${ }^{1,2}$ \\ ${ }^{1}$ CF-UM-UP, Centro de Física das Universidades do Minho e Porto, Departamento de Física, Universidade do Minho, Braga, Portuga \\ ${ }^{2}$ CBMA, Centro de Biologia Molecular e Ambiental, Departamento de Biologia, Universidade do Minho, Braga, Portugal \\ 3i3S, Instituto de Investigação e Inovação em Saúde e INEB, Instituto de Engenharia Biomédica, Universidade do Porto, Porto, Portugal \\ ${ }^{4} \mathrm{CESPU}$, Instituto de Investigação e Formação Avançada em Ciências e Tecnologias da Saúde, Instituto Universitário de Ciências da Saúde, Gandra, Portugal
}

*eduardabfer@gmail.com

\section{INTRODUCTION}

- The transport of drugs across cell membranes is a highly complex biological process involving the interaction of drugs with lipid barriers ${ }^{1}$. Numerous significant correlations between lipophilicity and membrane permeation have been stablished.
- Lipophilicity is the net result of all intermolecular forces, and when measured in the liposome/water systems, it also considers the ionic bounds, providing a better correlation with the intermolecular forces operating in molecular pharmacology and biochemistry².
Biomembrane mimetic models provide an alternative platform with very well defined and controlled conditions to help researchers from the drug discovery field to predict drugs' pharmacokinetic properties with therapeutic efficacy implications ${ }^{3,4}$.

\section{Log $P_{w / m}$ in Permeability: ACYCLOVIR}

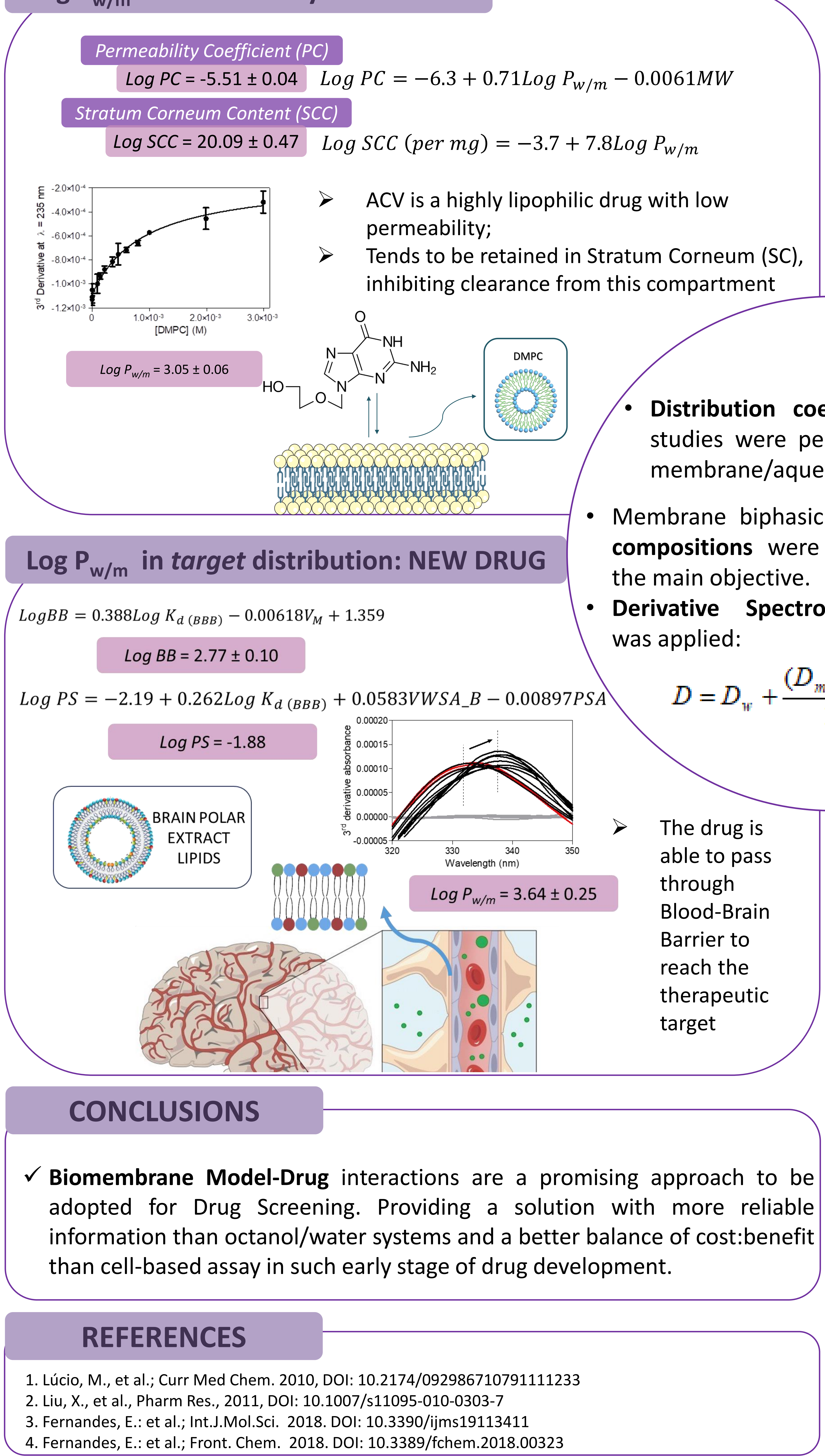

$\log \mathrm{P}_{\mathrm{w} / \mathrm{m}}$ in off-target Bioaccumulation: CAMPTOTHECIN MAIN BIOACCUMULATION SITES

$$
K_{\text {bioaccumulation }}=\frac{Q}{V P_{w / m}}
$$

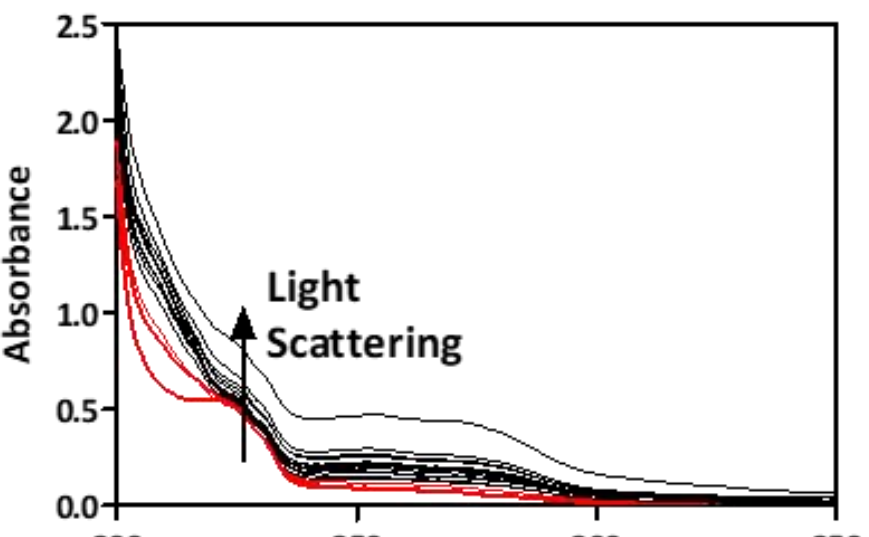

- $61 \%$ Adrenals $\quad \log P_{w / m}=3.14 \pm 0.13$

- $31 \%$ Thyroid

- $3 \%$ Heart (Basal)

- $3 \%$ Kidneys 